\title{
A Note on Connected Interior Domination in Join and Corona of Two Graphs
}

\author{
Leomarich F. Casinillo, Emily T. Lagumbay, and Hannah Rissah F. Abad \\ Department of Mathematics and Physics, College of Arts and Sciences, \\ Visayas State University, Baybay City, Leyte, Philippines
}

\begin{abstract}
Let $G$ be a non-complete connected graph of order greater or equal to 3 with vertex set $V(G)$ and edge set $E(G)$. An interior dominating set of $C \subseteq V(G)$ is called a connected interior dominating set of $G$ if the subgraphs $\langle C\rangle$ induced by $C$ is connected. The minimum cardinality of a connected interior dominating set of $G$, denoted by $\gamma_{\text {cId }}(G)$, is called the connected interior domination number. A connected interior dominating set of cardinality $\gamma_{\text {cId }}(G)$ is called a $\gamma_{c I d}-$ set of $G$. In this note, we revisit these concepts for some special graphs. Further, we characterize the connected interior dominating sets in the join and corona of two graphs and give some important results.
\end{abstract}

Keywords: Non-complete connected graphs, interior dominating set, connected interior dominating set, special graphs, join, corona

\section{Introduction}

Domination in graph provide numerous applications both in the position or location and protection strategies [1, 2]. This concepts was introduced by Claude Berge in 1958 [3, 5]. Also, Oystein Ore [4] introduced the terms dominating set and domination number in his book on graph theory which was published in 1962 . These concepts are helpful to find centrally located sets to cover the entire graph. The basics definitions and theorems used in this study can be found in $[2,6]$.

Let $G=(V(G), E(G))$ be a simple graph with vertex set $V(G)$ of finite order and edge set $E(G)$ and $v \in V(G)$. The neighborhood of $v$ is the set $N_{G}(v)=N(v)=\{u \in V(G): u v \in E(G)\}$. If $S \subseteq V(G)$, then the open neighborhood of $S$ is the set $N_{G}(S)=N(S)=\cup_{v \in S} N_{G}(v)$. The closed neighborhood of $S$ is $N_{G}[S]=$ $N[S]=S \cup N(S)$. The degree of a vertex $v$ in a graph $G$, denoted by $\operatorname{deg}_{G}(v)$, is the number of edges incident with $v$ in $G$. A leaf of graph $G$, is a vertex with degree 1 . A vertex that is a neighbor of a leaf is called a support. A cut vertex $v \in V(G)$ is a vertex that when removed from a graph $G$ creates more components than previously in the graph $G$.

Let $x$ and $z$ be two distinct vertices in $G$. The distance $d_{G}(x, z)$ between two vertices $x, z$ of a graph $G$ is defined as the length of the shortest walk between $\mathrm{u}$ and $\mathrm{v}$ in $\mathrm{G}$. If there is no walk between, $z$, then we declare $d_{G}(x, z)=\infty$. The eccentricity $e(v)$ of $v \in V(G)$ is the distance to a vertex farthest from $v$, that is, $e(v)=\max \left\{d_{G}(u, v): u \in V(G)\right\}$. The radius $r(G)$ of a graph $G$ is the minimum eccentricity of vertex in a graph $G$. A vertex $v \in V(G)$ is a central vertex if $e(G)=r(G)$. A vertex $y \in V(G)$ distinct from $x$ and $z$ is said to lie between $x$ and $z$ if $d_{G}(x, z)=d_{G}(x, y)+d_{G}(y, z)$. A vertex $v \in V(G)$ is an interior vertex of $G$ if for every vertex $u \in V(G)$ distinct from $v$, there exists a vertex $w \in V(G)$ such that $v$ lies between $u$ and $w$. A set $D \subseteq V(G)$ is a dominating set of $G$ if for every $v \in V(G) \backslash D$, there exists $u \in D$ such that $u v \in E(G)$, that is, $N_{G}[D]=V(G)$. A dominating set $I \subseteq V(G)$ is an interior dominating set if $I$ is dominating set of $G$ and every vertex $v \in I$ is an interior vertex of $G$. The interior domination number of $G$ is the minimum cardinality of an interior dominating set in $G$ and is denoted by $\gamma_{I d}(G)$. An interior dominating set of $C \subseteq V(G)$ is called a connected interior dominating set of $G$ if the subgraphs $\langle C\rangle$ induced by $C$ is connected. If $|C|=1$, then $C$ is a trivial connected interior dominating set. The minimum cardinality of a connected interior dominating set of $G$, denoted by $\gamma_{c I d}(G)$, is called the connected interior domination number. A connected interior dominating set of cardinality $\gamma_{c I d}(G)$ is called a $\gamma_{c I d}-$ set of $G$. The concepts of interior domination were introduced by Kinsley and Selvaraj [7] and connected domination by Sampathkumar and Walikar [8].

\section{Preliminary Results}

From the definitions, the following results are immediate.

Theorem 2.1. Let $G$ be a non-complete connected graph of order $n \geq 3$. Then,

$$
1 \leq \gamma_{I d}(G) \leq \gamma_{c I d}(G) \leq n-2
$$

Proof. Let $I \subseteq V(G)$ be a minimum interior dominating set of graph $G$. Then, $\gamma_{I d}(G)=|I|$. Let $C$ be a minimum connected interior dominating set of graph $G$, that is, $\gamma_{c I d}(G)=|C|$. If the elements of $I$ are 
connected interior vertices, then $|I|=|C|$. Otherwise $|I|<|C|$. Thus, $\gamma_{I d}(G) \leq \gamma_{c I d}(G)$. By definition of connected interior dominating set, then it is easy to check that $\gamma_{c l d}(G) \leq n-2$.

The upper bound in Theorem 2.1 is sharp and the lower bound is attainable.

Corollary 2.2. The difference $\gamma_{c I d}(G)-\gamma_{I d}(G)$ can be made arbitrarily large.

Theorem 2.3. Let $C$ be a $\gamma_{c I d}-$ set of a non-complete connected graph $G$ of order $n \geq 3$. Then,

i.) every cut-vertex is in $C$; and

ii.) every support is in $C$.

Proof. (i) Let $v$ be a cut-vertex in $G$. Suppose, for the sake of contradiction, that $v \notin C$. Since $G \backslash\{v\}$ is disconnected, then it is impossible to choose $C \subseteq G \backslash\{v\}$. Thus, every cut-vertex is in $C$.(ii). Note that every support vertex in $G$ is also a cut-vertex in $G$. Thus, every support is in $C$.

A leaf vertex of graph $\mathrm{G}$ will not be a member of interior vertex. In a tree graph, all vertices with degree greater than 1 will be a member of interior vertex.

Theorem 2.4. Let $G$ be a non-complete connected graph of order $n \geq 3$. Then, $\gamma_{c I d}(G)=1$ if and only if $G=K_{1, n}$.

Proof. Let $\gamma_{c l d}(G)=1$ and let $C=\{v\}$, where $v$ is the central vertex since $e(G)=r(G)$ and a trivial connected vertex that dominates every vertex in $G$. Also, an interior vertex in $G$. Thus, $G=K_{1, n}$. Conversely, suppose $G=K_{1, n}$. Then, clearly $\gamma_{c I d}(G)=1$.

Corollary 2.5: Let $G$ be a non-complete connected graph of order $n \geq 3$ with $|V(G)|=n$ and $|E(G)|=m$. If $\gamma_{c I d}(G)=1$, then $\sum_{v \in V(G) \backslash C} \operatorname{deg}(v)=2 m-n+1$.

The following remark is the result of connected interior domination number on some special graphs.

Remark 2.6. Let $n$ and $m$ be positive integers. Then,
i.) $\quad \gamma_{c I d}\left(P_{n}\right)=n-2 \quad$ where $n \geq 3$.
ii.) $\quad \gamma_{c I d}\left(C_{n}\right)=n-2 \quad$ where $n \geq 4$.
iii.) $\quad \gamma_{\text {cId }}\left(F_{n}\right)=1 \quad$ where $n \geq 3$
iv.) $\quad \gamma_{c I d}\left(W_{n}\right)=1 \quad$ where $n \geq 3$.
v.) $\quad \gamma_{\text {cId }}\left(K_{m, n}\right)=2 \quad$ where $m \geq 1$ and $n \geq 1$ but $m=n \neq 1$.

For any complete graph $K_{n}$, where $n \geq 3$, there is no connected interior dominating set, since for distinct vertices $x, y, z \in V\left(K_{n}\right), d_{G}(x, z) \neq d_{G}(x, y)+d_{G}(y, z)$.

\section{Connected Interior Domination In The Join Of Two Graphs}

The join of two graphs $G$ and $H$ is the graph $G+H$ with vertex-set $V(G+H)=V(G) \dot{\cup} V(H)$ and edge-set $E(G+H)=E(G) \dot{\cup} E(H) \cup\{u v: u \in V(G), v \in V(H)\}$.

Lemma 3.1. If $C$ is a connected interior dominating set of a graph $G$, then $C$ is a connected dominating set of $G$.

Proof. Suppose that $C$ is a connected interior dominating set of $G$. Then, $C$ is an interior dominating set of $G$, that is, $C$ is a dominating set of $G$. Now, let $u \in V(G) \backslash C$. Then, there exists $v \in C$ such that $u v \in E(G)$ for every $u \in V(G) \backslash C$ and the subgraph $\langle C\rangle \quad$ induced by $C$ is connected. Thus, $C$ is a connected dominating set of $G$.

The converse of Lemma 3.1 is not necessarily true. We need Lemma 3.1 for the next result.

Theorem 3.2. Let $G$ and $H$ be connected graphs of order $n \geq 3$ but not both complete. Then, $C \subset V(G+H)$ is a connected interior dominating set in $G+H$ if and only if one of the following two statements holds:

i.) $\quad C$ is a connected dominating set of $G$.

ii.) $\quad C$ is a connected dominating set of $H$.

Proof. Suppose that $C \subset V(G)$ or $C \subset V(H)$. If $C \subset V(G)$, then $C$ is a connected dominating set of $G$ by Lemma 3.1. Thus, (i) holds. Similarly by Lemma 3.1, (ii) holds.

For the converse, suppose the statement (i) holds. Since $C$ is a dominating set of $G$, then $C$ is a dominating set of $G+H$. Let $u, w \in V(G+H)$. Then, there exists $v \in C$ distinct for $u$ and $w$ such that $d_{G+H}(u, w)=$ 
$d_{G+H}(u, v)+d_{G+H}(v, w)$. Accordingly, $C$ is a connected interior dominating set of $G+H$ if (i) holds. In similar manner, if (ii) holds, then $C$ is a connected interior dominating set of $G+H$.

Our next result characterizes all non-complete graph $G$ of order $n \geq 3$ joined with any graph $H$.

Theorem 3.3. Let $G$ be a non-complete connected graph of order $n \geq 3$ and let $k$ be a positive integer. Then, $\gamma_{c I d}(G+H)=k$ if and only if $\gamma_{c I d}(G)=k$ for any graph $H$.

Proof. Suppose that $\gamma_{c I d}(G+H)=k$ and $C \subseteq V(G+H)$ where $|C|=k$. Let $v \in C \subseteq V(G)$. Then, for any graph $H, V(H) \subseteq N_{G}(v)$ for every $v \in C \subseteq V(G)$. Thus, $\gamma_{c I d}(G)=k$.

Conversely, suppose that $\gamma_{c l d}(G)=k$ and $C \subseteq V(G)$. Let $u \in C$. Then, $u v \in E(G+H)$ for every $v \in V(H)$. It follows that $u \in N_{H}(v)$ for every $v \in V(H)$. Thus, $\gamma_{c I d}(G+H)=k$.

Corollary 3.4. Let $G$ be non-complete connected graph of order $n \geq 3$. Then, $\gamma_{c I d}(G)=1$ if and only if $G \cong K_{1}+H$, for any non-complete graph $H$.

Theorem 3.5. Let $G$ be non-complete connected graph of order $n \geq 3$ and $H=K_{m}$, where $m \geq 2$. If $v \in C \subseteq$ $V(G)$ and $u \in V(H)$, then $(C \backslash\{v\}) \cup\{u\}$ is also dominating in $G+H$.

Proof. Suppose that $C \subseteq V(G)$ and $u \in V(H)$. Let $v \in C \subseteq V(G)$. Then, $v \in N_{H}(u)$ for every $u \in V(H)$ and $v \in C \subseteq V(G)$ is the dominating vertex in $H+v$ for every $v \in C$. Since $H$ is complete, then $H+v$ is also complete. It follows that $(C \backslash\{v\}) \cup\{u\}$ is dominating $\quad$ set in $G+H$.

Corollary 3.6. Let $G=K_{1}$ and $H=P_{n}$, where $n \geq 3$. Then, $\gamma_{c I d}(G+H)=|V(G)|=1$.

For $G=K_{m}$ and $H=K_{n}$, where $m, n \geq 1$, there is no connected interior dominating set for $G+H$, since no interior vertex in $G, H$, and $G+H$.

\section{Connected Interior Domination In The Corona Of Two Graphs}

Let $G$ and $H$ be graphs of order $m$ and $n$, respectively. The corona of two graphs $G$ and $H$ is the graph $G \circ H$ obtained by taking one copy of $G$ and $m$ copies of $H$, and then joining the $i t h$ vertex of $G$ to every vertex of the ith copy of $H$. For every $v \in V(G)$, denote by $H^{v}$ the copy of $H$ whose vertices are attached one by one to the vertex $v$. Denote by $v+H^{v}$ the subgraph of the corona $G \circ H$ corresponding to the join $\left\langle\{v\}+H^{v}\right\rangle$.

Theorem 4.1 [9] Let $G$ be connected graph and $H$ be any graph. Then, $D \subseteq V(G \circ H)$ is a dominating set of $G \circ H$ if and only if $V\left(v+H^{v}\right) \cap D$ is a dominating set of $v+H^{v}$ for every $v \in V(G)$.

We need Theorem 4.1 for the characterization of connected interior dominating sets in the corona of two graphs.

Theorem 4.2. Let $G$ be connected graph with order $n \geq 2$ and $H$ be any non-complete graph. Then, $C \subset V(G \circ$ $H$ ) is a connected interior dominating set of $G \circ H$ if and only if $C=V(G)$.

Proof. Suppose that $C \subset V(G \circ H)$ is a connected interior dominating set of $G \circ H$. Then, by Theorem 4.1, $C_{v}=C \cap V\left(v+H^{v}\right)$ is a dominating set of $v+H^{v}$ for every $v \in V(G)$. Since $\cup_{v \in V(G)} C_{v}=C$ is connected and $G$ is connected with order $n \geq 2$, then for every $u \in V(G)$ distinct from $v$, there exists $w \in V\left(H^{v}\right)$ such that $d_{G \circ H}(u, w)=d_{G \circ H}(u, v)+d_{G \circ H}(v, w)$ for every $v \in V(G)$. It follows that $C_{v}$ is interior dominating vertex in $v+H^{v}$. This implies that for every $w \in V\left(H^{v}\right), w \notin C_{v}$ for every $v \in V(G)$. Then, $C_{v}=\{v\}$ for every $v \in$ $V(G)$.

Thus, $\mathrm{\cup}_{v \in V(G)} C_{v}=C=V(G)$.

Conversely, suppose that $C=V(G)$ holds. Then, it follows that $C$ is connected since $G$ is connected with order $n \geq 2$. Let $C_{v}=\{v\}$ for every $v \in V(G)$. Then, for every $w \in V\left(H^{v}\right), v w \in E\left(v+H^{v}\right)$ and there exists vertex $u \in V(G)$ distinct from $v$ such that $d_{G \circ H}(u, w)=d_{G \circ H}(u, v)+d_{G \circ H}(v, w)$ for every $v \in V(G)$. Thus, $C=V(G)$ is a connected interior dominating set of $G \circ H$.

The next corollaries and remark are direct consequence of Theorem 4.2.

Corollary 4.3. Let $H$ be non-complete graph of order $n \geq 2$. Then, $\gamma_{c I d}(G+H)=1$ if and only if $G=K_{1}$. 
Proof. Let $C$ be a connected interior dominating set of $G \circ H$. Then, $C=V(G)$ is a connected interior dominating set of $G \circ H$ by Theorem 4.2. Suppose that $\gamma_{c I d}(G+H)=1$. Then, it follows that $|V(G)|=$ 1. Thus, $G=K_{1}$.

For the converse, let $v \in V(G)$ and $H$ be non-complete graph of order $n \geq 2$. Suppose that $G=K_{1}$. Then, $V(G)=\{v\}$ and there exists $u, w \in V\left(H^{v}\right)$ such that $d_{G \circ H}(u, w)=d_{G \circ H}(u, v)+d_{G \circ H}(v, w)$. Thus, it is immediate that $\gamma_{c I d}(G+H)=1$.

Corollary 4.4. Let $G$ be any connected graph with $|V(G)|=n \geq 2$ and $H$ be any non-complete graph with $|V(H)|=m \geq 2$. Then, $|V(G \circ H) \backslash C|=n m$.

Remark 4.5. Let $G=P_{n}$, where $n \geq 2$ and $H$ be any non-complete graph. Then, the following statements holds:
i.) $\quad \bigcap_{v \in V(G)} N_{G \circ H}\left(I_{v}\right)=\phi$.
ii.) $\quad \bigcup_{v \in V(G)} N_{G \circ H}\left(I_{v}\right)=V(G \circ H)$.

The next result deals with the security of dominating set of graph $G \circ H$, for any connected graph $G$ and $H=K_{n}$.

Theorem 4.6. Let $G$ be any connected graph and $H=K_{n}$, where $n \geq 2$. If $v \in C$ and $u \in V(G \circ H) \backslash C$, then $(C \backslash\{v\}) \cup\{u\}$ is a dominating set of $G \circ H$.

Proof. Suppose that $C \subset V(G \circ H)$. By Theorem 4.2, $C_{v}=C \cap V\left(v+H^{v}\right)$ and $\mathrm{U}_{v \in V(G)} C_{v}=C=V(G)$. By the definition of corona of two graphs, $v+H^{v}$ is complete since $H$ is complete. It follows that $C_{v}=\{v\}$ is the minimum dominating set of $v+H^{v}$. Let $u \in V\left(H^{v}\right)$ for every $v \in V(G)$. This implies that $\left(C_{v} \backslash\{v\}\right) \cup\{u\}$ is dominating set of $v+H^{v}$. Thus, $\cup_{v \in V(G)}\left[\left(C_{v} \backslash\{v\}\right) \cup\{u\}\right]=(C \backslash\{v\}) \cup\{u\}$ is a dominating set of $G \circ H$.

Corollary 4.6. Let $G=P_{2}$ and $H$ be any graph. Then, there exists $x, y \in C$ such that

$$
V(G \circ H) \backslash\{x, y\}=\left(N_{G \circ H}(x) \backslash\{y\}\right) \cup\left(N_{G \circ H}(y) \backslash\{x\}\right)
$$

It is worth noting that $\gamma_{c I d}(G \circ H)=2$, where $G=P_{2}$ and for any graph $H$.

\section{Conclusion}

In this note, we obtained the bounds of connected interior domination number for any graph $G$. We also determined the connected interior domination number of some special graphs and investigated the connected interior dominating set of join and corona of two graphs. Some of the results are characterization of join and corona of two graphs.

\section{Acknowledgements}

The authors would like to acknowledge the referee for the rigorous review and giving significant comments for the improvement of this paper.

\section{References}

[1]. E. C. Castillano and R. A. L. Ugbinada, Secure domination in the joins of graphs, Applied Mathematical Sciences, Vol. 8, 2014, 5203-5211.

[2]. T. W. Haynes, S. T. Hedetniemi, and P. J. Slater, Fundamentals of domination in graphs, Marcel Dekker, New York, 1998.

[3]. E. J. Cockayne, and S. T. Hedetniemi, Towards a theory of domination in graph, Networks, 1977, 247-261.

[4]. O. Ore, Theory of graphs. Amer. Mathe Soc. Colloq. Publication (Amer. Soc., Providence, RI), 1962

[5]. J. Tarr, Domination in graphs, University of South Florida, 2010, 34-36.

[6]. E. Delavina, R. Pepper and B. Waller, Lower bounds for the domination number, Discussiones Mathematicae Graph Theory, January 2010.

[7]. A. A. Kinsley and C. C. Selvaraj, A study on interior domination in graphs, IOSR Journal of Mathematics Vol. 12, 2016, 55-59.

[8]. E. Sampathkumar and H. B. Walikar, The connected domination of a graph, Math. Phys. Sci. 13, 1979, 607-613.

[9]. C. E. Go and S. R. Canoy Jr., Domination in the corona and join of graphs. International Mathematical Forum. 6, 2011, no. 16, 763-771. 